\title{
Severe adverse events during sirolimus "off label" therapy for vascular anomalies
}

\author{
Jochen Rössler ${ }^{1}$, Eulalia Baselga ${ }^{2}$, Victoria Davila ${ }^{2}$, Verónica Celis ${ }^{3}$, Andrea Diociaiuto ${ }^{4}$, \\ May El Hachem ${ }^{4}$, Sandrine Mestre ${ }^{5}$, Dario Häberli ${ }^{6}$, Aram Prokop ${ }^{7}$, Christof Hanke ${ }^{8}$, \\ Wolfgang Loichinger ${ }^{9}$, Isabelle Quere ${ }^{5}$, Iris Baumgartner ${ }^{6}$, Charlotte Niemeyer $^{10}$, and \\ Friedrich Kapp ${ }^{10}$ \\ ${ }^{1}$ Inselspital University Hospital Bern \\ ${ }^{2}$ Hospital Sant Joan de Deu \\ ${ }^{3}$ Hospital Sant Joan de Déu \\ ${ }^{4}$ Bambino Gesù Children Hospital, IRCCS \\ ${ }^{5}$ University Hospital of Montpellier, FAVA-MULTI Reference Centre for Lymphedema and \\ lymphatic anomalies, University of Montpellier \\ ${ }^{6}$ Swiss Cardiovascular Center, Inselspital, Bern University Hospital, University of Bern \\ ${ }^{7}$ Children's Hospital \\ ${ }^{8}$ Diakonie Klinikum \\ ${ }^{9}$ University Hospital of Ulm \\ ${ }^{10}$ University Medical Center Freiburg
}

June 22, 2020

\begin{abstract}
Objectives: Clinical studies have shown low toxicity and a favorable safety profile for sirolimus in vascular anomalies. Here, we describe severe adverse events (SAEs) observed during "off-label use". Methods: We performed a retrospective, multicenter chart review for SAEs during "off-label" sirolimus therapy for vascular anomalies and analyzed these cases by a pre-designed workflow. Results: We identified 17 SAEs in 14 patients diagnosed with generalized lymphatic anomaly (n=4), Gorham-Stout disease $(n=2)$, central conducting lymphatic anomaly $(n=1)$, lymphatic malformation $(n=4)$, tufted angioma $(n=1)$, kaposiform hemangioendothelioma $(n=1)$, and venous malformation in a CLOVES syndrome $(n=1)$. Three patients presented two SAEs. The age at initiation of sirolimus therapy was under the age of 2 years $(5 \mathrm{x}), 2$ to 6 years $(5 \mathrm{x})$ and older than 12 years (4x). SAEs occurred during the first 3 months of sirolimus therapy (7x), between 3 and 12 months $(7 x)$ and after one year of therapy (3x). The most frequent SAE was viral pneumonia (8x) resulting in death due to a metapneumovirus infection in a 3 months old and generalized adenovirus infection in a 28 months old child. Sirolimus blood level at the time of SAEs ranged between 2.7 and 21 ng/L. Five patients were on antibiotic prophylaxis during sirolimus therapy. Conclusions: Most SAEs are observed in the first year of sirolimus therapy; however, SAEs can also occur after a longer treatment period. SAEs are potentially life threatening, especially in early infancy. Presence risk factors, i.e. underlying vascular anomaly or immune status, may contribute to the risk of SAEs.
\end{abstract}

\section{Introduction}

In the last years, sirolimus, a well-established mTOR inhibitor, has entered the therapeutic field of vascular anomalies [1] and is currently administered for complicated vascular tumors, such as kaposiform hemagioendothelioma (KHE), as well as for vascular malformations refractory to standard therapy, including 
venous malformations (VM) and lymphatic malformations (LM). Initial observations of the effectiveness of sirolimus lead to a small case series [2] followed by a clinical study in the US that included all types of vascular anomalies refractory to previous therapy [3]. Several other studies followed analyzing the effect of sirolimus in defined subgroups of vascular anomalies, such as VM, LM [4], generalized lymphatic anomalies (GLA) [5], Gorham Stout disease (GSD) [5], blue rubber bleb nevus syndrome (BRBNS) [6] or in KHE with Kasabach-Merritt phenomenon (KMP) [7]. Taken together, the therapeutic benefit of sirolimus could clearly be demonstrated, although complete remission as a therapeutic end point was never reached.

In parallel to the clinical use of sirolimus, genetic testing has elucidated the molecular mechanism of the activity of the mTOR inhibitor in vascular anomalies. Somatic and germline mutations in the tyrosinekinase transmembrane receptor TEK were found in VM [8], while the presence of PI3KCA mutations could be demonstrated in tissue of VM and LM [9] as well as in endothelial cells of LM [10]. Furthermore, PI3KCA mutations have been described in overgrowth tissue leading to the description of the PI3KCA Related Overgrowth Spectrum (PROS) [11]. Both PIK3CA and TEK have thus been identified [8] as a therapeutic targets and are located upstream of mTOR in its signaling pathway.

Sirolimus has been used for many years as an immunosuppressive agent in patients after organ transplantation, a population at high risk for opportunistic infections. In patients with vascular anomalies, the safety profile of the drug appears very favorable. However, two reports with infectious complications due to sirolimus in this patient population can be found in the literature: the first report is on a child with KHE and KMP who developed pneumocystis jirovecii pneumonia (PJP) while on sirolimus and a prednisolone taper [12]. The child recovered under therapy with trimethoprim-sulfamethoxazole (TMP-SMX). The second report is on two infants with KHE and KMP who both manifested paroxysmal cough and tachypnea shortly after the onset of sirolimus treatment and finally died [13]. Authors of both manuscripts advice to administer prophylactic trimethoprim/sulfamethoxazole (TMP-SMX) for the prevention of pneumocystis jirovecii pneumonia (PJP) as implemented in the US clinical trial. Furthermore, a trial with sirolimus for the treatment of PROS-patients showed a surprisingly high incidence of severe adverse events (SAE with $>$ grade 3 severity in $26 \%$ of patients), including infectious complications [14].

Here, we report the results of a survey on the nature of SAE in patients treated with sirolimus in an off-label setting.

\section{Methods}

We performed a retrospective chart review from September 2019 to April 2020 at two European Reference Centers for Vascular Anomalies (Freiburg/Germany, and Rome/Italy), three national referral centers for Vascular Anomalies (Barcelona/Spain, Montpellier/France and Bern/Switzerland) and two German Pediatric Oncology units (Köln and Ulm) to identify patients treated with sirolimus that developed an SAE. Medical history of the identified patients were analyzed using a pre-designed algorithm. Definition of SAE was according to Good Clinical Practice (GCP) rules and included any event that resulted in death, was life-threatening, required inpatient hospitalization or caused prolongation of existing hospitalization, resulted in persistent or significant disability/incapacity, might have caused a congenital anomaly/birth defect, or required intervention to prevent permanent impairment or damage. The treating physician had received patient/parental informed consent before transferring data for the study. This study is covered by the ethical committee numbers Freiburg 310/14 and 464/19.

\section{Results}

In this retrospective chart review, 7 centers reported on 17 SAEs in 14 patients, 3 patients had two SAEs. 4 patients were treated for GLA, 2 for GSD, 1 for CCLA, 4 for LM, 1 for a tufted angioma, 1 for a KHE with KMP and 1 for a VM in CLOVES (Table 1). The age at initiation of sirolimus therapy was younger than 2 years $(6 \mathrm{x}), 2$ to 6 years $(4 \mathrm{x})$ and older than 12 years $(4 \mathrm{x})$.

From 14 patients sirolimus blood level results at the timepoint of SAEs were available and ranged between 2.7 and $21 \mathrm{ng} / \mathrm{L}$. "Low-dose" sirolimus resulting in a target plasma concentration of $2-6.9 \mathrm{ng} / \mathrm{ml}$ [14] was 
administered in 8 (57\%) patients, "high-dose" with 7.0-15.0 ng/ml in $6(43 \%)$ patients. One patient (P12) exceeded the recommended dosing resulting in elevated sirolimus levels. Five patients received anti-infectious prophylaxis: 4 patients TMP-SMX and 1 child penicillin. Eight of the $17(47 \%)$ SAEs occurred during the first 3 months of sirolimus therapy, additional 6 SAEs (35\%) were observed between 3 and 12 months. Three SAE $(18 \%)$ occurred after more than 1 year on sirolimus.

The most frequent SAE, 8 of 17 instances (47\%), was respiratory infection demanding hospitalization and oxygen therapy. In 5 of such respiratory SAEs, the infectious agent could be identified: rhinovirus/enterovirus (P01, P12), HHV7 virus (P12), metapneumovirus (P02, P06), parainfluenca virus (P06), adenovirus (P03) and pneumococcus (P12) could be isolated. Pneumococcus was also responsible for a sepsis with meningitis in one of these patients (P03). In three patients, SAE were due to an infection of a foreign body, such as a port-a-cath system (P04) or osteosynthesis material stabilizing a pathological fracture in two GSD patients (P08,P13). A HSV-infection and paralytic ileus occurred after cessation of sirolimus during intensive care treatment (P14). Finally, an accompanying local candida albicans infection proven by buccal swap was found (P12).

The type of underlying vascular anomaly appeared to put the patient at a certain risk for specific SAEs. A GLA patient with a paraspinal soft tissue lesion (P11) experienced claudication and loss of balance, and a VM patient (P07) thrombophlebitis. Two episodes with severe diarrhea as consecutive SAEs (P09) and one episode with an ileus (P03) were reported, all in patients with a GLA and an underlying bowel involvement.

All except three SAEs (P02, P03, P14) were completely resolved after 3 to 15 days of hospitalization, P14 had a partial recovery after 2 months and could be weaned from the tracheostomy but needed non-invasive ventilation at night. Nine patients continued while 5 patients discontinued the drug after the first SAE, 1 patient (P09) stopped after the second SAE. Notably, the patient with KHE and KMP (P04) experiencing 2 SAEs continued sirolimus even after the second SAE.

Two SAEs resulted in death of the patients. The first was a 3 months old child with a microcystic LM in the face (P02) who had a metapneumovirus infection 2 months after start of sirolimus and died immediately 4 days later. The second fatal SAE was observed in a 28 months old child with protein losing enteropathy in the context of GLA (P03) and an acute ileus accompanied by an adenovirus respiratory infection. Three days after hospitalization, the child succumbed to the infection. Importantly, the child was under a penicillin prophylaxis, which had been initiated after a first SAE at the age of 14 months for a pneumococcus infection 2 weeks into sirolimus therapy. It cannot be excluded that these patients in addition suffered from an underlying immunodeficiency that rendered them more susceptible to severe infections.

\section{Discussion}

Sirolimus is a relatively old drug, that has successfully been used as an immunosuppressive agent in the context of solid organ transplantation [15]. The possibility to measure sirolimus serum trough levels makes the therapy well controllable. There is, however, no recommendation for an effective and safe serum level for pediatric patients [16]. Furthermore, data on safety of sirolimus therapy in children for its use in the approved indications is scarce. In a clinical study on children with autoimmune lymphoproliferative syndrome, no major side effects in the 30 patients with a median age of 11 years treated with sirolimus were reported [17].

In the context of vascular anomalies, sirolimus has been administered for the last several years. In the phase II trial from the U.S. for patients with complicated vascular anomalies 57 patients were treated with a median age of 8.1 years at start of therapy [3]. The most common toxicities (grades 3 or 4) attributed to sirolimus included blood/bone marrow (27\% of patients), metabolic/laboratory (3\%), gastrointestinal $(3 \%)$, infectious $(2 \%)$, lymphatic $(2 \%)$, and/or pulmonary/upper respiratory (2\%) complications. Dose reduction was required in 2 patients, one associated with possible laryngospasm and the second related to hypertriglyceridemia. Two patients were taken off study secondary to toxicity: One patient had persistent grade 2 nausea and the second grade 3 lymphedema. No toxicity-related deaths were observed. Interestingly, no SAEs were reported in 6 patients reported from the University Hospital in Graz, Austria, who had started sirolimus therapy under the age of 2 years ( $4 \mathrm{x}$ at birth, and at 10 and 13 months) and continued therapy for 
3 to 56 months [18].

Most of the SAEs in this survey were infections that could be managed by hospitalization, antibiotics and supportive care. The most frequent cause of the respiratory infections were viruses demonstrating that a prophylactic antibiotic therapy during sirolimus cannot mitigate the risk of all infectious complications. Unfortunately, results on immune status (lymphocytes, immunoglobuline level, etc.) from the affected patients were not available. Risk factors of the underlying vascular malformations appear important, e.g. in GSD osteosynthesis material in fractured bones can easily be infected. Furthermore, lung and/or bowel involvement as well as spleen involvement may be a predisposing factor for severe infections, because most patients with SAEs in this survey suffered from LM and especially complex lymphatic anomalies such as GLA and GSD.

There are 3 fatal cases of SAEs during sirolimus therapy in the literature: The first case is due to pneumocystis jirovecii pneumonia (PJP) leading to respiratory failure, which required extracorporeal membrane oxygenation. Importantly, sirolimus was administered in combination with steroids, which might have contributed to the development of PJP [12]. The other two fatal cases had respiratory failure as well; however, an infectious agent could not be identified [13]. Additionally, Parker et al. reported SAEs in a pilot study on 39 patients with PROS and progressive overgrowth treated with sirolimus [14]: 23 SAEs occurred among 12 participants of the study leading to discontinuation of sirolimus in seven patients. The most common class of adverse effects (AEs) was infection in $41 \%$ patients, followed by blood or lymphatic disorders in $21 \%$. Clinically important AEs included grade 4 neutropenia (neutrophil count $0.02 \times 10^{9} / \mathrm{L}$ ), interstitial pneumonitis, and a sirolimus hypersensitivity syndrome with prolonged fever. Sirolimus was withdrawn in all three participants, all of whom subsequently made full recoveries.

Most SAE reported in our retrospective survey were observed during the first year of sirolimus therapy. However, some patients experienced SAE with sirolimus therapy after several years. Two SAEs in our cohort lead to death in young infants. Presence of further risk factors, i.e. of the underlying vascular anomaly could play a role, as it was the case in the GLA patient with lung and bowl involvement (P03). Viral infections like metapneumovirus, rhinovirus, parainfluenca viurs and adenovirus must be added to the list of possible opportunistic infections next to the already known risk for PJP.

The major limitation of this retrospective study is the "off-label" setting. There was no harmonized treatment protocol, and "low-dose" as well as "high-dose" sirolimus has been administered. Interestingly, the occurrence of SAEs did not seem to be dependent on the sirolimus blood level. Furthermore, anti-infectious prophylactic measures were not standardized. Moreover, we cannot calculate the true frequency of SAEs during sirolimus therapy from our data because the number of patients with vascular anomalies treated with sirolimus cannot accurately be estimated, since some patients for whom sirolimus treatment was recommended then received therapy at another hospital. Calculating the incidence of SAEs of sirolimus with the number of patients treated with sirolimus at the participating centers would thus overestimated the true incidence. In addition, it would be helpful to have more detailed information on the immune status of the reported patients to exclude underlying risk factors.

Nevertheless, we suggest that patients treated with sirolimus for vascular lesion may be at a significant risk for severe adverse events and should be monitored carefully, even when they are maintained at a low sirolimus trough levels. Furthermore, all recommended vaccinations should be realized before start of sirolimus therapy and dead vaccines should be continued to be administered according to recommendations during sirolimus therapy. Finally, experienced investigators should only administer sirolimus for vascular anomalies, preferably in the setting of a clinical study.

Most recently, inhibitors of PI3KCA are under clinical evaluation in patients with vascular anomalies. A first case series of 20 patients showed preliminary results with efficacy and few mild side effects [19]. Hopefully, this more selective therapeutic approach will provide better therapy for patients affected with these rare and heterogeneous disorders.

\section{Conclusion}


Sirolimus still is a new treatment option for vascular anomalies that has shown to provide some benefit to patients, especially with LM and VM. The safety profile has generally been favorable, although different patient populations might be more prone to complications. The results of our retrospective survey of the "off-label" use of sirolimus in different vascular anomalies illustrates the risk of SAEs including fatal outcomes in these patients. This highlights the importance of a close follow-up in these patients as well as the need for further clinical studies to establish the safety profile of sirolimus, the incidence of SAEs in different patient populations and the optimal antibiotic prophylaxis.

\section{Conflict of Interest}

Dr. Rossler reports personal fees from SOBI, Roche and Bayer, all for Advisory Board membership, and all outside the submitted work. Dr. Rossler and Dr. El Hachem report consulting fees from Pierre Fabre outside the submitted work. Dr. Niemeyer receives consulting fees from Novartis and BMS, all outside the submitted work. Dr. Kapp reports consulting fees from Novartis outside the submitted work. All other authors declare no competing interests.

\section{Acknowledgments}

Five of the authors of this publication are members of the Vascular Anomalies Working Group (VASCA WG) of the European Reference Network for Rare Multisystemic Vascular Diseases (VASCERN) - Project ID: 769036. This work was supported in part by the Center for Vascular Anomalies, Freiburg Center for Rare Diseases. F.G.K. was supported by an EXCEL-Fellowship of the Faculty of Medicine, University of Freiburg, funded by the Else-Kroner-Fresenius-Stiftung.

\section{References}

1. Sandbank S, Molho-Pessach V, Farkas A, Barzilai A, Greenberger S:Oral and Topical Sirolimus for Vascular Anomalies: A Multicentre Study and Review . Acta dermato-venereologica 2019,99 (11):990-996.

2. Hammill AM, Wentzel M, Gupta A, Nelson S, Lucky A, Elluru R, Dasgupta R, Azizkhan RG, Adams DM: Sirolimus for the treatment of complicated vascular anomalies in children. Pediatric blood E cancer 2011, 57 (6):1018-1024.

3. Adams DM, Trenor CC, 3rd, Hammill AM, Vinks AA, Patel MN, Chaudry G, Wentzel MS, MobberleySchuman PS, Campbell LM, Brookbank C et al :Efficacy and Safety of Sirolimus in the Treatment of Complicated Vascular Anomalies . Pediatrics 2016, 137 (2):e20153257.

4. Hammer J, Seront E, Duez S, Dupont S, Van Damme A, Schmitz S, Hoyoux C, Chopinet C, Clapuyt P, Hammer $\mathrm{F}$ et al : Sirolimus is efficacious in treatment for extensive and/or complex slow-flow vascular malformations: a monocentric prospective phase II study. Orphanet journal of rare diseases 2018, 13 (1):191.

5. Ricci KW, Hammill AM, Mobberley-Schuman P, Nelson SC, Blatt J, Bender JLG, McCuaig CC, Synakiewicz A, Frieden IJ, Adams DM: Efficacy of systemic sirolimus in the treatment of generalized lymphatic anomaly and Gorham-Stout disease . Pediatric blood \& cancer 2019,66 (5):e27614.

6. Wong XL, Phan K, Rodriguez Bandera AI, Sebaratnam DF:Sirolimus in blue rubber bleb naevus syndrome: A systematic review . Journal of paediatrics and child health 2019,55 (2):152-155.

7. Wang Z, Yao W, Sun H, Dong K, Ma Y, Chen L, Zheng S, Li K:Sirolimus therapy for kaposiform hemangioendothelioma with long-term follow-up . The Journal of dermatology 2019,46 (11):956-961.

8. Limaye N, Kangas J, Mendola A, Godfraind C, Schlogel MJ, Helaers R, Eklund L, Boon LM, Vikkula M: Somatic Activating PIK3CA Mutations Cause Venous Malformation . Am J Hum Genet 2015,97 (6):914-921. 
9. Luks VL, Kamitaki N, Vivero MP, Uller W, Rab R, Bovee JV, Rialon KL, Guevara CJ, Alomari AI, Greene AK et al : Lymphatic and other vascular malformative/overgrowth disorders are caused by somatic mutations in PIK3CA . The Journal of pediatrics 2015,166 (4):1048-1054 e1041-1045.

10. Blesinger H, Kaulfuss S, Aung T, Schwoch S, Prantl L, Rossler J, Wilting J, Becker J: PIK3CA mutations are specifically localized to lymphatic endothelial cells of lymphatic malformations . PLoS One 2018, 13 (7):e0200343.

11. Venot Q, Canaud G: [PIK3CA-related overgrowth syndrome (PROS)] . Nephrologie \& therapeutique 2017, 13 Suppl 1 :S155-S156.

12. Russell TB, Rinker EK, Dillingham CS, Givner LB, McLean TW:Pneumocystis Jirovecii Pneumonia During Sirolimus Therapy for Kaposiform Hemangioendothelioma . Pediatrics 2018,141 (Suppl 5):S421-S424.

13. Ying H, Qiao C, Yang X, Lin X: A Case Report of 2 Sirolimus-Related Deaths Among Infants With Kaposiform Hemangioendotheliomas . Pediatrics 2018, 141 (Suppl 5):S425-S429.

14. Parker VER, Keppler-Noreuil KM, Faivre L, Luu M, Oden NL, De Silva L, Sapp JC, Andrews K, Bardou M, Chen KY et al : Safety and efficacy of low-dose sirolimus in the PIK3CA-related overgrowth spectrum . Genetics in medicine : official journal of the American College of Medical Genetics 2019, 21 (5):1189-1198.

15. Sindhi R: Sirolimus in pediatric transplant recipients .Transplantation proceedings 2003, 35 (3 Suppl):113S-114S.

16. Roque EJ, Rios MG, Vignolo AP, Pinochet VC, Schultz M, Humeres AR, Delucchi A, Rius AM, Hepp KJ: [Use of Sirolimus in five pediatric patients undergoing solid organ transplantation] .Revista medica de Chile 2008, 136 (5):631-636.

17. Bride KL, Vincent T, Smith-Whitley K, Lambert MP, Bleesing JJ, Seif AE, Manno CS, Casper J, Grupp SA, Teachey DT: Sirolimus is effective in relapsed/refractory autoimmune cytopenias: results of a prospective multi-institutional trial . Blood 2016,127 (1):17-28.

18. Lackner H, Karastaneva A, Schwinger W, Benesch M, Sovinz P, Seidel M, Sperl D, Lanz S, Haxhija $\mathrm{E}$, Reiterer $\mathrm{F}$ et al :Sirolimus for the treatment of children with various complicated vascular anomalies . European journal of pediatrics 2015,174 (12):1579-1584.

19. Venot Q, Blanc T, Rabia SH, Berteloot L, Ladraa S, Duong JP, Blanc E, Johnson SC, Hoguin C, Boccara O et al : Targeted therapy in patients with PIK3CA-related overgrowth syndrome .Nature 2018, 558 (7711):540-546.

Hosted file

5_PBC_Table.docx available at https://authorea.com/users/335017/articles/460949-severeadverse-events-during-sirolimus-off-label-therapy-for-vascular-anomalies 